\title{
A Multichannel Superconducting Tunnel Junction Detector for High-Resolution X-Ray Spectroscopy of Magnesium Diboride Films
}

\author{
Stephan Friedrich, Arturas Vailionis, Owen Drury, Thomas Niedermayr, Tobias Funk, W. N. Kang, Eun-Mi Choi, \\ Hyeong-Jin Kim, Sung-Ik Lee, Stephen P. Cramer, Changyoung Kim, and Simon E. Labov
}

\begin{abstract}
We have built a high-resolution soft x-ray spectrometer using a $3 \times 3$ array of superconducting $\mathrm{Nb}$-Al-AlOx-Al-Nb tunnel junctions. The detector is cooled to $\sim 0.1 \mathrm{~K}$ by a two-stage adiabatic demagnetization refrigerator while being held at the end of a 40-cm-long detector snout that can be inserted into an experimental chamber. The $0.6 \mathrm{~mm} \times 0.6 \mathrm{~mm}$ array has an energy resolution of 10-15 eV FWHM for $x$-ray energies below $1 \mathrm{keV}$ and total count rate capabilities above 100000 counts/s. We demonstrate the capabilities of the instrument in a study of anisotropy in $c$-axis oriented $\mathrm{MgB}_{2}$ films by near edge $\mathrm{x}$-ray absorption spectroscopy. The absorption fine structure at the $B \mathrm{~K}$-edge differs for excitation with $\mathrm{x}$-rays polarized along the $c$-axis and in the $a b$-plane of the $\mathrm{MgB}_{2}$ film. We discuss the implications of the anisotropy for the density of states in $\mathrm{MgB}_{2}$ at the Fermi surface.
\end{abstract}

Index Terms - Josephson junctions, magnesium diboride, superconducting devices, X-ray spectrometers.

\section{INTRODUCTION}

O UPERCONDUCTING tunnel junctions (STJ) have been developed over the last decade as fast, high-resolution energy-dispersive X-ray detectors [1]. Their operation is based on measuring the increase in tunneling current after an X-ray absorbed in one of the superconducting electrodes creates excess quasiparticles in proportion to the X-ray energy $\mathrm{E}_{x}$. STJ's offer a higher energy resolution than conventional $\mathrm{Ge}$ or $\mathrm{Si}(\mathrm{Li})$ detectors because the average energy $\varepsilon(\varepsilon \approx 1.7 \Delta)$ to create an excess quasiparticle scales with the superconducting energy gap $\Delta$ and

Manuscript received August 5, 2002. This work was supported in part by the National Science Foundation under Grant DMR-0114216 and by the National Institutes of Health under Grant GM-44380. This work was performed under the auspices of the U.S. Department of Energy by University of California Lawrence Livermore National Laboratory under Contract W-7405-Eng-48.

S. Friedrich and O. Drury are with the Advanced Detector Group at Lawrence Livermore National Laboratory, Livermore, CA 94550 USA and also with the University of California, Davis, CA 95616 USA (e-mail: friedrich1@1lnl.gov).

A. Vailionis is with the Geballe Laboratory for Advanced Materials, Stanford University, Stanford, CA 94305 USA (e-mail: arturas@stanford.edu).

T. Niedermayr and S. E. Labov are with the Advanced Detector Group at Lawrence Livermore National Laboratory, Livermore, CA 94550 USA.

T. Funk is with the Physical Biosciences Division at Lawrence Berkeley National Laboratory, Berkeley, CA 94720 USA.

W. N. Kang, E.-M. Choi, H.-J. Kim, and S.-I. Lee are with the National Creative Research Initiative Center for Superconductivity, Pohang University of Science and Technology, Pohang 790-784, Korea (e-mail: silee@ postech.ac.kr). S. P. Cramer is with the Physical Biosciences Division at Lawrence Berkeley National Laboratory, Berkeley, CA 94720 USA and is also with the University of California, Davis, CA 95616 USA (e-mail: spcramer@lbl.gov).

C. Kim is with the Department of Physics at Yonsei University, Seoul

120-749, Korea (e-mail: cykim@ phya.yonsei.ac.kr).

Digital Object Identifier 10.1109/TASC.2003.814169 is thus about three orders of magnitude smaller than in semiconductors. The full-width at half-maximum (FWHM) energy resolution $\Delta E_{F W H M}$ of STJ detectors is ultimately limited by the statistical fluctuations in the quasiparticle generation and tunneling processes:

$$
\Delta E_{F W H M}=2.355 \sqrt{\varepsilon E_{x}(F+1+1 /\langle n\rangle)} .
$$

Here $F \approx 0.2$ is the Fano factor that quantifies the statistical fluctuations during quasiparticle generation and $\langle n\rangle$ is the average number of tunneling events each quasiparticle undergoes [2]-[4]. For large $\langle n\rangle$, the energy resolution of $\mathrm{Nb}$ or Ta-based STJ detectors can theoretically range from $\sim 1 \mathrm{eV}$ to $\sim 10 \mathrm{eV}$ FWHM for X-ray energies between 0.1 and $10 \mathrm{keV}$. STJ's therefore offer a competitive advantage in X-ray fluorescence experiments whenever conventional semiconductor detectors lack energy resolution and grating spectrometers lack detection efficiency to collect data within an acceptable period of time [5], [6].

The Advanced Detector Group at Lawrence Livermore National Laboratory has developed STJ detectors for x-ray spectroscopy in material science, biophysics, astronomy and national security applications [6], [7]. Our material science and biophysics research is done in collaboration with the University of California Davis and the Lawrence Berkeley National Lab at the Advanced Light Source synchrotron. For these applications, we have designed a soft X-ray spectrometer that allows operating STJ detector arrays inside an experimental chamber at the end of a synchrotron beam line.

Here we summarize the present performance of the spectrometer for synchrotron-based fluorescence-detected absorption spectroscopy. As an example, we present soft X-ray absorption spectra on the boron K-edges of $c$-axis oriented $\mathrm{MgB}_{2}$ films. We observe difference in the absorption signal for excitation in the $a b$-plane and excitation along the $c$-axis of the film, and discuss these results in terms of the orbitals involved in the chemical bonding.

\section{SPECTROMETER CHARACTERIZATION}

\section{A. Superconducting Tunnel Junction Array}

The STJ detectors we use for spectroscopy at the synchrotron consist of Nb-Al-AlOx-Al-Nb thin film structures with a 265 $\mathrm{nm} \mathrm{Nb}$ base electrode, an Al-AlOx-Al tunnel junction whose $50 \mathrm{~nm} \mathrm{Al}$ films serve as quasiparticle traps, and a $165 \mathrm{~nm}$ top 
$\mathrm{Nb}$ absorber film. We currently operate $3 \times 3$ arrays of $200 \mu \mathrm{m}$ $\times 200 \mu \mathrm{m}$ STJ devices. The $\mathrm{Nb}$ base electrode is common to all nine pixels such that the entire array can be read out with only 10 wires.

$\mathrm{X}$-rays absorbed in the top $\mathrm{Nb}$ film excite excess quasiparticles in proportion to the X-ray energy, which diffuse into the adjacent $\mathrm{Al}$ film and are trapped by inelastic scattering. As they tunnel through the AlOx barrier, they produce a current pulse that is directly read out with a FET-based preamplifier at room temperature [8]. Our STJ's can be operated at temperatures below $0.4 \mathrm{~K}$ without temperature regulation.

The nine preamplifier signals are processed with commercial Canberra 2026 amplifiers, digitized by a 8071 analog-to-digital converter and captured on a DEC Alpha workstation. The acquisition program also controls the beam line computer to scan the energy of the incident beam and to adjust its polarization.

\section{B. Adiabatic Demagnetization Refrigerator Design}

Adiabatic demagnetization is a process of magnetic cooling below a liquid He bath temperature through isothermal magnetization and adiabatic demagnetization of a paramagnetic material. Magnetization lowers the entropy of the paramagnet, and the heat of magnetization is carried into the liquid He bath through a closed heat switch. After opening the heat switch, the magnetic field is decreased sufficiently slowly to keep the entropy of the paramagnet constant, thereby lowering its temperature.

The adiabatic demagnetization refrigerator (ADR) used in these experiments employs two thermally isolated stages with two different paramagnets that are magnetized simultaneously inside the same $5 \mathrm{~T}$ magnet bore (Fig. 1) [9]. The first stage uses a gallium gadolinium garnet $\mathrm{Gd}_{3} \mathrm{Ga}_{5} \mathrm{O}_{12}$ (GGG) to attain a temperature of $\sim 1 \mathrm{~K}$. It supports a second stage with iron ammonium sulfate $\mathrm{Fe}\left(\mathrm{NH}_{4}\right)\left(\mathrm{SO}_{4}\right)_{2} \times 12 \mathrm{H}_{2} 0$ (FAA, for ferric ammonium alum) that holds the detector array at a temperature of $\sim 0.1$ $\mathrm{K}$. The two-stage design allows operation with a bath temperature of $4.2 \mathrm{~K}$ and thus does not require pumping on the liquid $\mathrm{He}$ tank. The cryostat achieves a base temperature of $70 \mathrm{mK}$, and a hold time per magnetization cycle of $\sim 20$ hours below $0.4 \mathrm{~K}$, the maximum operating temperature of our STJ's. It is compact, easy to use and functions reliably during weeklong beam times.

For fluorescence experiments, the ADR has a cold finger that extends $40 \mathrm{~cm}$ outside the cryostat wall into an experimental chamber [10]. The STJ detector array is mounted at the end of an oxygen-free high-conductivity $\mathrm{Cu}$ rod that is bolted to the $0.1 \mathrm{~K}$ stage at three points for stability and thermal contact. This rod is surrounded by a liquid-He-cooled $\mathrm{Cu}$ radiation shield that also holds the STJ detector magnet needed to suppress the dc Josephson current and Fiske mode resonances for stable STJ operation. All of this is enclosed with a second radiation shield attached to the liquid- $\mathrm{N}_{2}$-cooled stage. The diameter of this outer shield of about $45 \mathrm{~mm}$ is sufficiently small to fit through standard gate valves. All of these parts are Au plated to prevent them from oxidation for good thermal contact across interfaces.

There are three thin infrared (IR) blocking windows at the end of the cold finger to prevent room temperature radiation from heating the cold stage and causing IR induced excess noise in the detector, one at $77 \mathrm{~K}$, one at $4.2 \mathrm{~K}$ and one on the cold stage

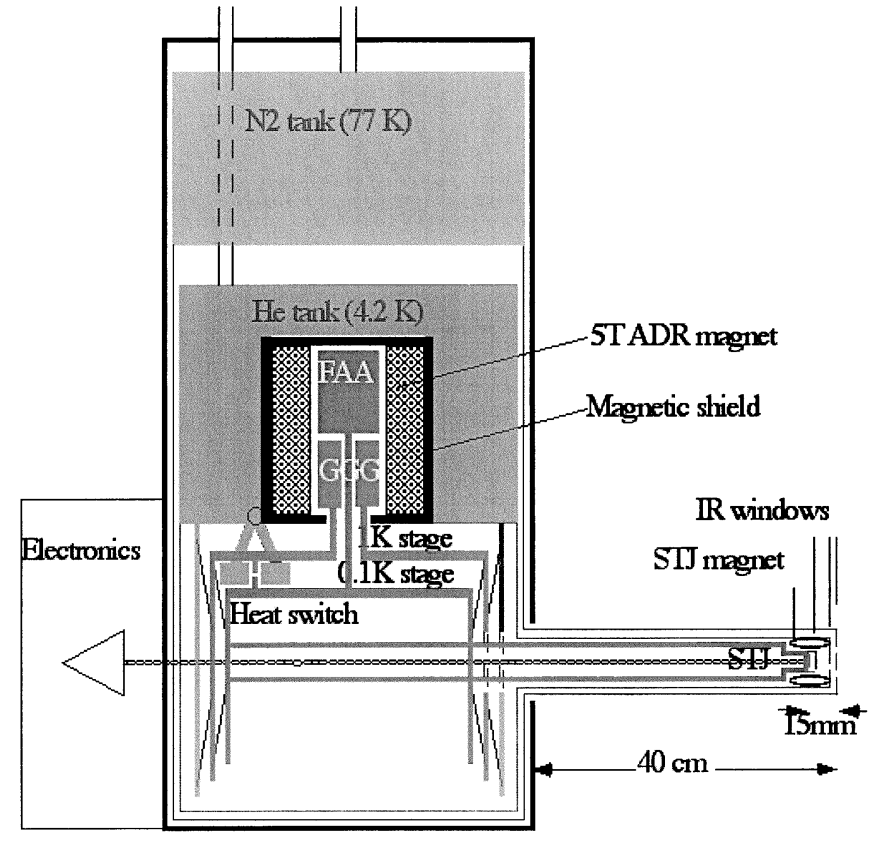

Fig. 1. Schematic of the two-stage adiabatic demagnetization refrigerator. The STJ detector array is held at a temperature of $\sim 0.1 \mathrm{~K}$ the end of a $40-\mathrm{cm}$-long cold finger within $\sim 20 \mathrm{~mm}$ of a room temperature sample.

at $0.1 \mathrm{~K}$ directly in front of the detector array. Their size and their thickness is determined by a trade-off between desired angle of acceptance and tolerable IR photon flux. For soft X-ray spectroscopy below $500 \mathrm{eV}$ we use IR blocking windows consisting of $200 \AA \mathrm{Al}$ on $1000 \AA$ parylene on a 95\% transmissive $\mathrm{Cu}$ grid.

It is important that the cryostat be UHV compatible to prevent residual gases from freezing out on the IR windows and the detector array. Since ADR's cannot be baked out (the FAA salt pill decomposes above $\sim 40^{\circ} \mathrm{C}$ ), we are using no superinsulation, use only vented screws, and have assembled the cryostat under clean room conditions. We can operate the ADR in the low $10^{-9}$ Torr pressure range and have observed no decrease in detection efficiency due to gas freeze-out on the IR windows during weeks of operation.

\section{Detector Performance}

We have characterized the STJ detector performance by illuminating the devices directly with a monochromatic synchrotron beam. Fig. 2 shows the measured resolution of a 100 $\mu \mathrm{m} \times 100 \mu \mathrm{m} \mathrm{STJ}$ detector held at the end of the cold finger in the energy range between 0.1 and $1 \mathrm{keV}$ (solid circles). The resolution varies between 6.8 and $9.5 \mathrm{eV}$ FWHM in this energy range. This is somewhat less than the energy resolution of 1.7 to $8.9 \mathrm{eV}$ FWHM measured with nominally identical devices operated deep inside an ADR [11], [12]. The discrepancy is mostly due to increased electronic noise from a finite IR photon transmission through the comparably large solid angle subtended by the 3 and $5 \mathrm{~mm}$ diameter IR blocking windows right in front of the detector. The degradation in resolution in the range between 100 and $200 \mathrm{eV}$ arises from the fact that the top $\mathrm{Nb}$ film is partially transparent in this energy region. Photons are thus absorbed both in the $\mathrm{Nb}$ absorber and the $\mathrm{Al}$ trap underneath where the gap is smaller, and the difference 


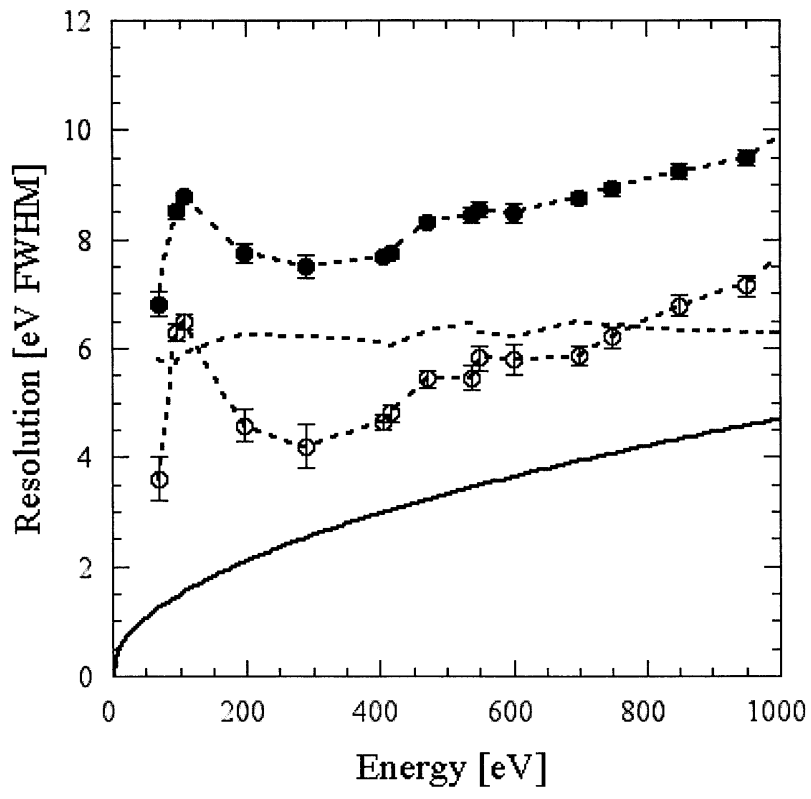

Fig. 2. Energy resolution for a single pixel detector as a function of X-ray energy. The intrinsic detector resolution (open circles) is obtained by subtracting the electronic noise (dashed line) from the measured resolution (solid circles). It approaches the fundamental resolution limit of our detectors set by the statistics of quasiparticle generation and tunneling according to (1) for $\langle n\rangle \approx 3.1$ (solid line).

in signal amplitude leads to a broadening of the observed line [13].

We emphasize that the energy resolution of the $200 \mu \mathrm{m} \times$ $200 \mu \mathrm{m}$ STJ's is not quite as good as the data shown in Fig. 2. Larger devices have larger capacitance and higher leakage currents, both of which increase the electronic contribution to the line broadening. Furthermore, spatial inhomogeneities tend to have a bigger impact for larger devices. However, for the experiments on $\mathrm{MgB}_{2}$ discussed here it is desirable to trade-off some of the resolution for an increased detection efficiency. We therefore choose to operate arrays of $200 \mu \mathrm{m} \times 200 \mu \mathrm{m}$ STJ's whose resolution is $\sim 15 \mathrm{eV}$ FWHM.

For smaller high-resolution devices, we have also measured the resolution as a function of count rate by varying the exit slit of the beam line and thereby the incident photon flux (Fig. 3). The X-ray energy was held constant at $E_{x}=277 \mathrm{eV}$ corresponding to the energy of carbon K fluorescence. No pile-up rejection was used in these experiments, so the numbers quoted are the actual throughput per detector. For count rates below 10000 counts/s, we can use the optimum shaping time of $4 \mu \mathrm{s}$ and observe a constant resolution of $\sim 8.5 \mathrm{eV} \mathrm{FWHM.} \mathrm{At} \mathrm{a}$ count rate of 22000 counts/s pile has started to degrade the energy resolution to $10.2 \mathrm{eV}$.

For count rates much higher than 22000 counts/s, the shaping amplifier saturates with $4 \mu$ s shaping and a shorter shaping time must be used. This reduces the energy resolution, but allows higher throughput. For a shaping time of $1 \mu \mathrm{s}$, the resolution at 43000 counts/s is $19.3 \mathrm{eV}$ FWHM, and for applications where an energy resolution around $40 \mathrm{eV}$ is sufficient, we operate a single STJ up to 100000 counts/s with $0.25 \mu \mathrm{s}$ shaping. The entire 9-channel array thus has a total count rate

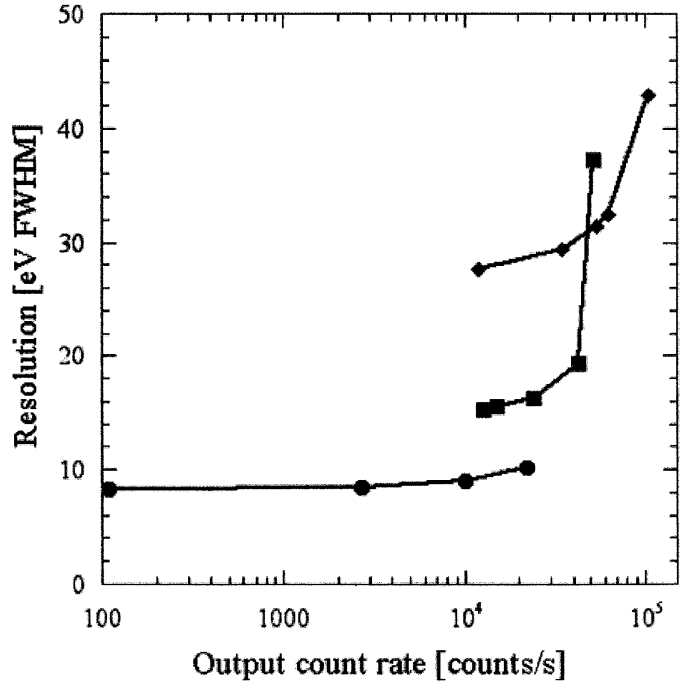

Fig. 3. Energy resolution as a function of count rate at a photon energy $E_{x}=$ $277 \mathrm{eV}$ for three different shaping times. The three curves show the trade-off between slow $4 \mu$ s shaping (circles), intermediate $1 \mu$ s shaping (squares) and fast $0.25 \mu$ s shaping (diamonds).

capability around 100000 counts/s at the highest resolution, and significantly above that with reduced resolution.

\section{Matching STJ Spectrometer-Synchrotron Flux}

A modern undulator beam line at a third generation synchrotron like the Advanced Light Source produces a monochromatic soft X-ray beam with a flux of order $10^{12}$ photons/s. Fluorescence yields in the soft X-ray range, i.e., for the $\mathrm{K}$ fluorescence of light elements or for transition metal $\mathrm{L}$ lines, are typically around $10^{-3}$. There is thus a total X-ray fluorescence of $10^{9}$ photons/s emitted into $4 \pi$. Our STJ detector array with a total area of $0.6 \mathrm{~mm} \times 0.6 \mathrm{~mm}$ covers a solid angle $\Omega / 4 \pi \approx 10^{-4}$. The photon flux for the array is thus around $10^{5}$ photons/s, and each of the STJ pixels is typically operated at $10^{4}$ counts/s. The capabilities of the superconducting spectrometer are thus well matched to the flux of modern soft x-ray beam lines.

\section{SOFT X-RAY SPECTROMETRY}

\section{A. Motivation}

The recent discovery of superconductivity in $\mathrm{MgB}_{2}$ with a transition temperature of $39 \mathrm{~K}$ has sparked considerable scientific and technological interest in this material [14]. Although its transition temperature is lower than that of the CuO-based high- $\mathrm{T}_{c}$ superconductors, it is high enough to be easily accessible by mechanical cryocoolers. Furthermore, $\mathrm{MgB}_{2}$ has a comparably simple crystal structure, which raises the hope that long sheets or wires with high critical currents might be more easily fabricated from $\mathrm{MgB}_{2}$ than from CuO-based high- $\mathrm{T}_{c}$ materials. The boron atoms in $\mathrm{MgB}_{2}$ form hexagonal prisms, with $\mathrm{sp}_{2}$-hybridized $\sim 3.5 \AA$ diameter honeycomb lattices in the $a b$-plane, and a $c$-spacing between planes of about $3.5 \AA$ A. The intraplanar B-B bonds are much shorter than the distance between the planes, and hence the B-B bonding is 
strongly anisotropic. The $\mathrm{Mg}$ donates two electrons to $\mathrm{B}$, and superconductivity is thought to occur mostly in the metal-like $\mathrm{B}$-sheets [15]. One question is if the layered structure of $\mathrm{MgB}_{2}$ leads to an anisotropy in the density of states, and consequently an anisotropy in the current carrying capabilities of individual $\mathrm{MgB}_{2}$ grains.

As an example for high-resolution synchrotron-based X-ray spectroscopy with our STJ detector array, we present measurements of the anisotropy of the X-ray absorption fine structure at the B K-edge in oriented $\mathrm{MgB}_{2}$ films. A more detailed analysis of the anisotropy in the density of states in terms of the $\mathrm{MgB}_{2}$ band structure will be published in a forthcoming paper.

\section{B. Fluorescence-Detected Absorption Spectroscopy}

Synchrotron-based x-ray absorption spectroscopy (XAS) samples atomic energy levels with sub-eV resolution by scanning the energy of a monochromatic beam through an absorption edge of the element of interest. For concentrated samples, the absorption spectrum of the element of interest is typically obtained from the intensity ratio of transmitted to incident beam (transmission mode), or by electron yield measurements. For dilute samples, the sensitivity can be enhanced by recording the intensity of the corresponding x-ray fluorescence as a measure of absorption (fluorescence-detected XAS). This greatly reduces the number of background counts, provided that the fluorescence line of interest can be separated from neighboring lines in multi-element specimens [16]. Fluorescence-detected XAS is also preferred in experiments where electron yield measurements are likely to produce artifacts. This occurs in samples whose surface may have been oxidized, or in samples which show a decrease in electron yield because of resonant enhancement of the fluorescence yield. Both of these effects are of concern in $\mathrm{MgB}_{2}$. We therefore follow the approach by Callcott et al. and analyze the $\mathrm{MgB}_{2}$ films by fluorescence-detected XAS [17].

\section{Sample Preparation and Characterization}

The $\mathrm{MgB}_{2}$ films were fabricated at the National Creative Research Initiative Center for Superconductivity at the Pohang University of Science and Technology, Korea. They are grown by depositing an amorphous $\mathrm{B}$ film on an $\mathrm{Al}_{2} \mathrm{O}_{3}$ substrate using pulsed laser deposition, sealing it in a $\mathrm{Nb}$ tube in $\mathrm{Ar}$ atmosphere together with high purity $\mathrm{Mg}$ metal, and heat treating it at $900{ }^{\circ} \mathrm{C}$ for 10-30 $\mathrm{min}$ in an evacuated quartz ampoule. Details of the sample preparation are described elsewhere [18].

The films have a critical temperature of $39 \mathrm{~K}$ with a sharp transition of $\sim 0.2 \mathrm{~K}$ width. The film thickness is $\sim 4000 \AA$. $\mathrm{X}$-ray $\Theta-2 \Theta$ diffraction measurements show that the $\mathrm{MgB}_{2}$ film has a highly $c$-axis oriented crystal structure normal to the substrate surface (Fig. 4). The film's lattice constant of $3.496 \AA$ is somewhat smaller than the $3.522 \AA$ characteristic for bulk $\mathrm{MgB}_{2}$, because it is grown on $\mathrm{Al}_{2} \mathrm{O}_{3}$ with a 3.481 A lattice constant. Different $c$-axis oriented grains are oriented randomly in the $a b$-plane of the film [19].

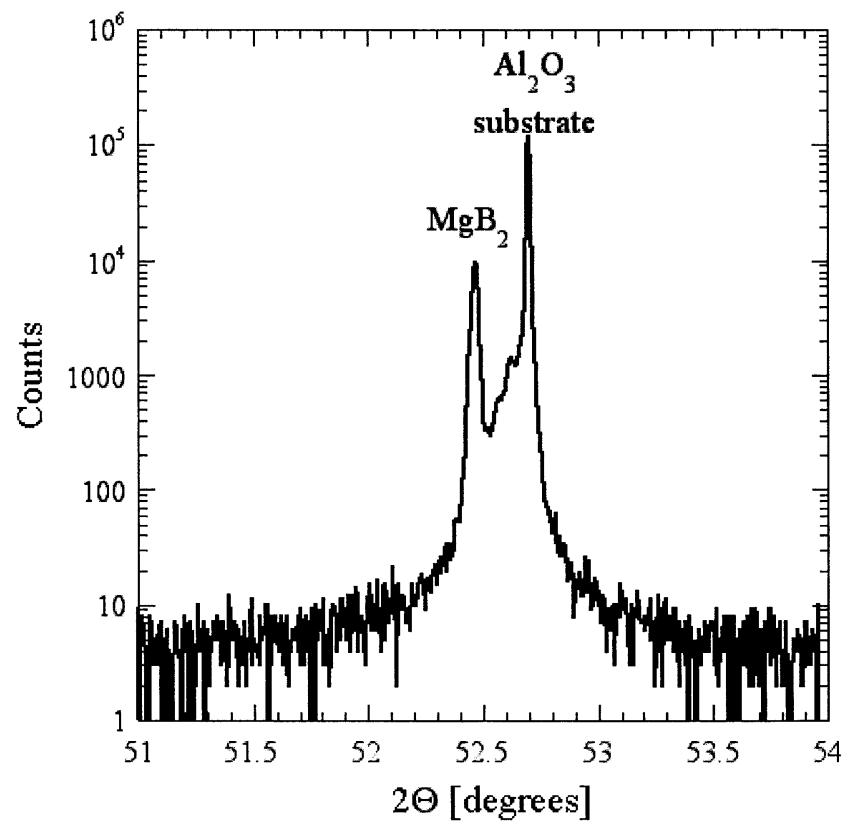

Fig. 4. X-ray $\Theta-2 \Theta$ diffraction scans showing a highly $c$-axis oriented crystal structure of the $\mathrm{MgB}_{2}$ film despite a small lattice mismatch between $\mathrm{MgB}_{2}$ and the $\mathrm{Al}_{2} \mathrm{O}_{3}$ substrate.

\section{Near Edge X-Ray Absorption Spectroscopy}

The soft X-ray spectroscopy was performed at beam line 4.0.2 at the Advanced Light Source (ALS) synchrotron at Lawrence Berkeley National Laboratory. The sample was mounted to the base of a rotating sample holder in our UHV chamber at $\sim 10^{-8}$ Torr. The sample holder is attached to a He flow-cryostat that allows sample cooling from room temperature to $\sim 15 \mathrm{~K}$.

The X-ray absorption spectra are taken by scanning the energy of the incident synchrotron beam through the B K absorption edge, i.e., from 180 to $230 \mathrm{eV}$, and recording the resulting number of boron K fluorescence photons at $186 \mathrm{eV}$ with the STJ detector array. Since this number is proportional to the number of 1s holes generated and thus to the number of 1s photons excited into the boron $2 \mathrm{p}$ levels, the B fluorescence intensity is proportional to the number of empty states in the B $2 p$ levels. In cases where the matrix element of the $1 \mathrm{~s} \rightarrow 2 \mathrm{p}$ transition is constant and that the fluorescence yield is isotropic, the absorption signal provides a direct measure of the density of states above the Fermi energy at the boron sites.

In the energy range between 185 and $188 \mathrm{eV}$ around the Fermi energy we use a small step size of $0.1 \mathrm{eV}$, so that the absorption spectra are limited by the $0.1 \mathrm{eV}$ width of the incident beam. Between 188 and $195 \mathrm{eV}$ we use a step size of $0.2 \mathrm{eV}$, and in the other energy ranges the step size is $0.4 \mathrm{eV}$. The data acquisition time at each incident energy is $10 \mathrm{~s}$. We normalize the number of B partial fluorescence yield by the incident flux, subtract a small ( $\sim 2 \%$ of peak signal) constant background due to higher order excitation, and normalize the spectra to unity above $225 \mathrm{eV}$.

We select a horizontally polarized incident beam. The anisotropy of the absorption can then be measured by rotating the sample and comparing the signals for near normal $\left(80^{\circ}\right)$ or grazing incidence $\left(15^{\circ}\right)$ angle. To ensure that the observed differences are in fact due to anisotropic absorption rather than 


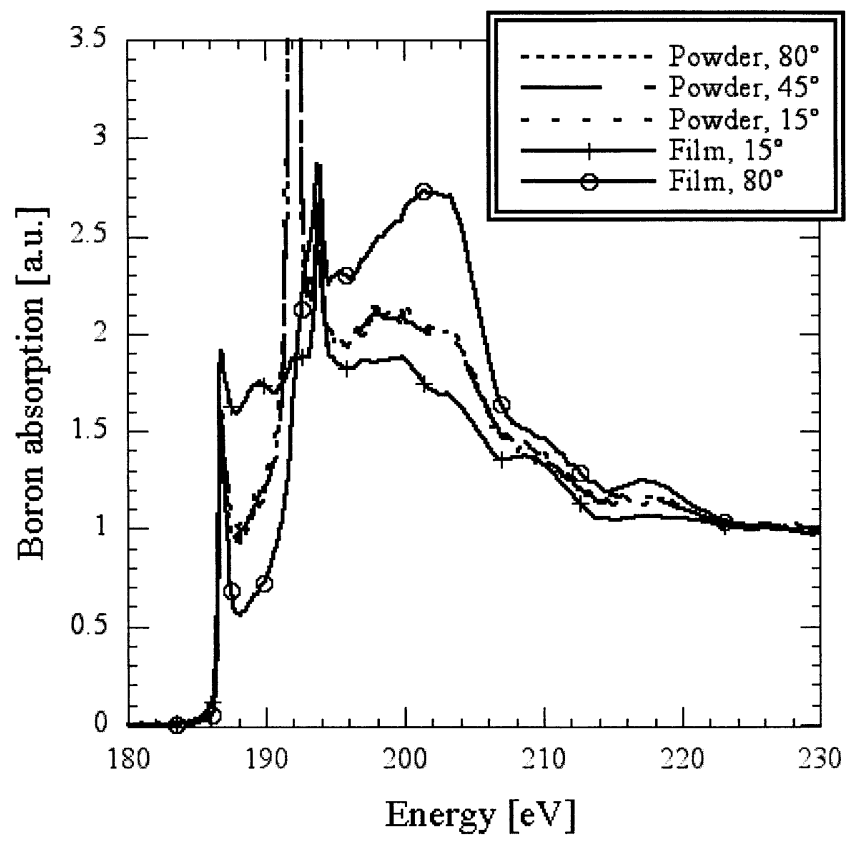

Fig. 5. Boron K-edge absorption spectra of the $\mathrm{MgB}_{2}$ film for $a b$-plane excitation (solid, with circles) and for excitation along the $c$-axis of the film (solid, with crosses). As expected, absorption spectra of the $\mathrm{MgB}_{2}$ powder (dotted) show no angle dependence and lie between the spectra for $a b$-plane and $c$-axis excitation. Note strong elastic peak at $192 \mathrm{eV}$ in powder spectra.

a measurement artifact, we also perform the same experiment on commercial $\mathrm{MgB}_{2}$ powder. This powder was re-ground in an inert atmosphere just before the measurement to produce fresh sample surfaces and minimize the effects of sample oxidation. To understand which spectral features are still due to sample oxidation (both the powder and the film had been kept in ambient air for several months), we also took absorption spectra on commercial $\mathrm{B}_{2} \mathrm{O}_{3}$ powder for comparison [20].

The resulting fluorescence-detected $\mathrm{X}$-ray absorption spectra are shown in Fig. 5. We observe a sharp increase in the absorption signal at the energy of $186.5 \mathrm{eV}$ corresponding to a high density of states at the Fermi level in $\mathrm{MgB}_{2}$. Above the Fermi energy, there is a clear difference between in-plane (incident angle $=80^{\circ}$ ) and out-of-plane (incident angle $=15^{\circ}$ ) absorption as expected from the strongly anisotropic $\mathrm{B}$ bonds. It is reassuring that the $\mathrm{MgB}_{2}$ spectra from the $\mathrm{MgB}_{2}$ powder taken under identical conditions do not show any angular dependence (dotted lines), and that the spectra lie between the two extreme cases of in-plane and out-of-plane excitation.

Note that the $\mathrm{MgB}_{2}$ powder spectra show a strong resonant line at $192 \mathrm{eV}$ that is not present in the film spectra. This structure is due to increased elastic scattering of the incident beam through an excitonic resonance [17]. We believe that the film spectra do not show this scattered elastic peak, because the smooth film surface will reflect the incident beam onto the small STJ detector only if the incident angle is exactly $45^{\circ}$, while there will always be grains in the powder for which this condition is fulfilled.

Comparison with the $\mathrm{B}_{2} \mathrm{O}_{3}$ absorption spectra (not shown) explains the peak at $194 \mathrm{eV}$ as due to sample oxidation [17]. The relative strength of the $\mathrm{B}_{2} \mathrm{O}_{3}$ signal is comparable in the powder and the film, since the sampling depth of the fluorescence signal

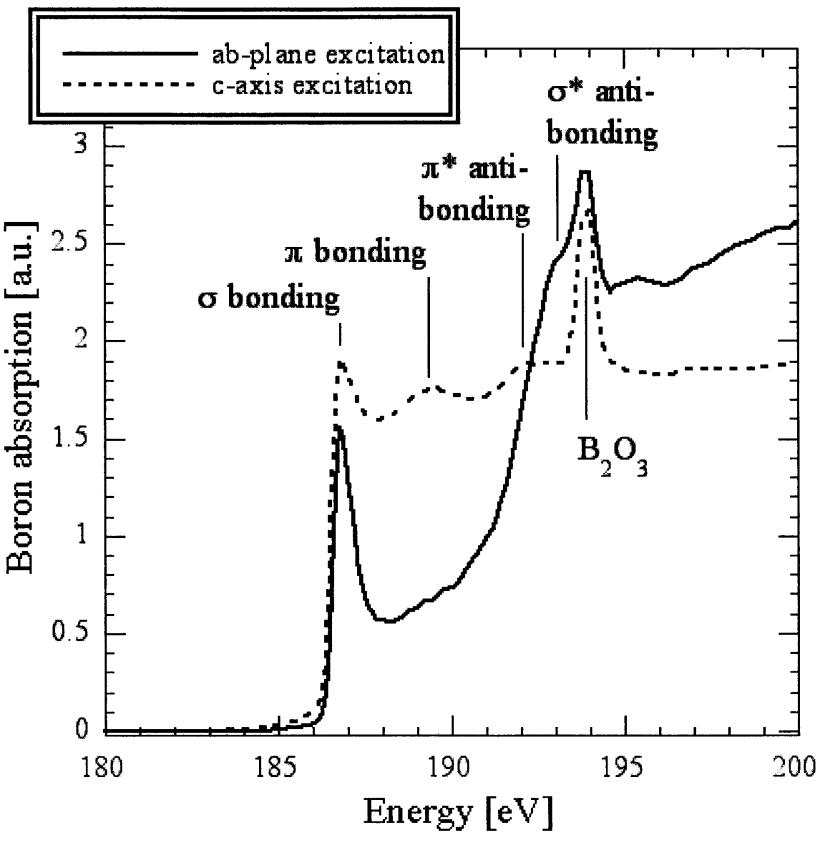

Fig. 6. Near edge absorption fine structure measured for in-plane excitation (solid) and out-of-plane excitation (dashed) of the $\mathrm{MgB}_{2}$ film. The different peaks correspond to empty $\sigma$ and $\pi$ bonding and anti-bonding molecular orbitals. $\sigma$ bonds lie in the $a b$-plane, while $\pi$ bonds lie along the crystal $c$-axis. The peak at $194 \mathrm{eV}$ is due to oxidation of the sample surface.

is bigger than the thickness of the oxidized surface layer formed after re-grinding the $\mathrm{MgB}_{2}$ powder.

\section{E. Discussion}

We now discuss the X-ray absorption fine structure as shown in Fig. 6 in terms of molecular orbitals. The boron $2 \mathrm{~s}, 2 \mathrm{p}_{x}$ and $2 \mathrm{p}_{y}$ orbitals in $\mathrm{MgB}_{2}$ are $\mathrm{sp}_{2}$ hybridized. These three $\mathrm{sp}_{2}$ orbitals form a planar structure with an angle of $120^{\circ}$ between their main lobes and give rise to the honeycomb structure in the $a b$-plane. If two lobes from neighboring atoms with different signs of the wavefunction overlap, they form a strong $\sigma$-bonding molecular orbital. The same lobes from a strongly antibonding $\sigma^{*}$ molecular orbital if their wavefunctions have the same sign. $\sigma$ bonds lie in the $a b$-plane and form the most stable lowest energy, and $\sigma^{*}$ the highest energy molecular orbitals. On the other hand, the $p_{z}$ orbitals are oriented perpendicular to the $a b$-plane in direction of the crystal $c$-axis. They form molecular $\pi$-bonding and $\pi^{*}$-antibonding orbitals. Since the overlap of their wavefunctions is not as strong as it is in the case of $\sigma$-bonds, the energy of the $\pi$-bonding orbitals is not as low, and the energy of the $\pi^{*}$ orbitals is not as high as that of their $\sigma$-bonding counterparts.

This can be seen in the X-ray absorption fine structure in Fig. 6. $\mathrm{Mg}$ donates electrons to the B sheets, so the lowest lying $\mathrm{sp}_{2} \sigma$-bonding orbitals are almost filled [15]. X-ray absorption occurs first into the empty parts of the $\sigma$-bonding orbitals, and it occurs predominantly for $a b$-plane excitation. This is consistent with calculations showing that the bands at the Fermi level derive mainly from $B$ orbitals [15]. The absorption features at 189 and $192 \mathrm{eV}$, which are much more prominent for $c$-axis excitation, correspond to transitions into the $\pi$ bonding and antibonding orbitals. Absorption into the highest-energy $\sigma^{*}$-anti- 
bonding molecular orbitals at $193 \mathrm{eV}$ partially overlaps with absorption by $\mathrm{B}_{2} \mathrm{O}_{3}$ formed at the sample surface. The nominally $a b$-plane and $c$-axis spectra show a small spectral contribution from the orthogonal component, because the maximum possible angles of $15^{\circ}$ and $80^{\circ}$ in our experiment do not allow perfect alignment of the beam polarization with the crystal axes.

The peak X-ray absorption signals at the Fermi energy have a ratio of $1: 0.82$ for excitation along the $c$-axis and in the $a b$-plane, respectively. To relate this ratio to the anisotropy of the density of states, we have to make the assumptions that the matrix element for the $1 \mathrm{~s} \rightarrow 2 \mathrm{p}$ transition must be the same for the different $\mathrm{p}$ orbitals, and that the B K fluorescence yield must be isotropic. Furthermore, we have to account for the fact that X-rays only sample electronic states in direction of the $\mathrm{X}$-ray polarization. Since the individual $c$-axis oriented grains are randomly oriented in the $a b$-plane, the in-plane absorption signal underestimates the density of states by a factor of 1.57 in the $a b$-plane. Under these assumptions we find that the ratio of the density of states $n_{c}: n_{a b}=1: 1.28$, i.e., the partial density of states in the $a b$-plane is roughly $30 \%$ higher than along the $c$-axis of the film. A relatively homogeneous density of states at the Fermi surface, together with a coherence length much larger than the layer spacing $\left(\xi_{c}=30 \AA, \xi_{a b}=37 \AA\right.$ in nominally identical films [21]) suggests that $\mathrm{MgB}_{2}$ has fairly isotropic electrical characteristics [15].

\section{SUMMARY}

We have built a high-resolution soft $X$-ray spectrometer based on a $3 \times 3$ array of $\mathrm{Nb}-\mathrm{Al}-\mathrm{AlOx}-\mathrm{Al}-\mathrm{Nb}$ superconducting tunnel junctions. It has an energy resolution of $\sim 15 \mathrm{eV}$ FWHM for $\mathrm{X}$-ray energies below $1 \mathrm{keV}$, and it can be operated at count rates above 100000 counts/s. The array is cooled to its operating temperature below $0.4 \mathrm{~K}$ by a two-stage adiabatic demagnetization refrigerator while being held at the end of a 40-cm-long cold finger that can be inserted into an experimental chamber.

We have used this spectrometer to study the anisotropy of the density of states in superconducting $\mathrm{MgB}_{2}$ films by fluorescence-detected absorption spectroscopy. We observe differences for absorption in the $a b$-plane and along the $c$-axis of the film, and explain them in terms of $\sigma$ and $\pi$ molecular bonding orbitals. Assuming a constant matrix element for $1 \mathrm{~s} \rightarrow 2 \mathrm{p}$ excitation and isotropic fluorescence yield, the partial density of states is found to be $\sim 30 \%$ higher in the $a b$-plane than along the $c$-axis of the $\mathrm{MgB}_{2}$ film. This suggests fairly isotropic electrical behavior.

These measurements demonstrate the capabilities of STJ spectrometers for synchrotron-based high-resolution soft X-ray spectroscopy.

\section{ACKNOWLEDGMENT}

The authors would like to thank J. D. Batteux for his excellent work in cryostat design, and E. Arenholz and A. T. Young for assistance with the beam line operation.

\section{REFERENCES}

[1] For a recent overview of the field of cryogenic X-ray detectors, see, Proceedings of the 9th International Workshop on Low Temperature Detectors, AIP Conference Proceedings, F. S. Porter, D. McCammon, M. Galeazzi, and C. K. Stahle, Eds., 2002, vol. 605.

[2] N. Rando et al., Nucl. Inst. Meth., vol. 313, pp. 173-176, 1992.

[3] C. A. Mears, S. E. Labov, and A. T. Barfknecht, Appl. Phys. Lett., vol. 63, pp. 2961-2963, 1993.

[4] D. J. Goldie et al., Appl. Phys. Lett., vol. 64, pp. 3169-3171, 1994.

[5] S. Kraft et al., J. Appl. Phys., vol. 86, pp. 7189-7191.

[6] M. Frank et al., Rev. Sci. Inst., vol. 69, pp. 25-31, 1998.

[7] S. E. Labov et al., Proc. of the 7th Intl. Workshop on Low Temperature Detectors, 1997, LTD-7, pp. 82-95.

[8] S. Friedrich et al., IEEE Trans. Appl. Superconductivity, vol. 7, pp. 3383-3386, 1997.

[9] C. Hagmann and P. L. Richards, Cryogenics, vol. 34, pp. 221-225, 1994.

[10] S. Friedrich et al., Nucl. Inst. Meth A, vol. 447/448, pp. 1117-1120, 2001.

[11] J. B. LeGrand, Appl. Phys. Lett., vol. 73, pp. 1295-1297, 1998.

[12] S. Friedrich, J. B. le Grand, L. J. Hiller, J. Kipp, M. Frank, S. E. Labov, S. P. Cramer, and A. T. Barfknecht, IEEE Trans. Appl. Superconductivity, vol. 9, pp. 3330-3333, 1999.

[13] S. Friedrich, L. J. Hiller, M. F. Cunningham, and S. E. Labov, IEEE Trans. Appl. Superconductivity, vol. 11, pp. 836-839, 2001.

[14] J. Nagamatsu, N. Nakagawa, T. Muranaka, Y. Zenitani, and J. Akimitsu, Nature, vol. 410, pp. 63-64, 2001.

[15] J. Kortus, I. I. Mazin, K. D. Belashchenko, V. P. Antropov, and L. L. Boyer, Phys. Rev. Lett., vol. 86, pp. 4656-4659, 2001.

[16] J. Jaclevic, J. A. Kirby, M. P. Klein, G. S. Brown, and P. Eisenberger, Solid State Comm., vol. 23, pp. 679-682, 1977.

[17] T. A. Calcott et al., Phys. Rev. B, vol. 64, pp. 132 504-132 506, 2001.

[18] W. N. Kang, E.-M. Choi, H.-J. Kim, and S.-I. Lee, Science, vol. 292, pp. $1521-1522,2001$

[19] H.-J. Kim, W. N. Kang, E.-M. Choi, M.-S. Kim, K. H. P. Kim, and S.-I. Lee, Phys. Rev. Lett., vol. 87, p. $087002,2001$.

[20] D. K. Aswal et al., Physica C, vol. 363, pp. 208-214, 2001.

[21] M. H. Jung et al., Chem. Phys. Lett., vol. 343, pp. 447-451, 2001. 